American Journal of Infectious Diseases 1 (2): 79-83, 2005

ISSN 1553-6203

(c) 2005 Science Publications

\title{
Granulomatous Amoebic Encephalitis: Clinical Diagnosis and Management
}

\author{
Naveed Ahmed Khan \\ School of Biological and Chemical Sciences, Birkbeck College, University of London \\ London, England, UK
}

\begin{abstract}
Granulomatous amoebic encephalitis (GAE) is a serious human disease with fatal consequences. With the mortality rate of more than $90 \%$, it is not surprising that the majority of GAE infections are identified at the post-mortem stage. The most distressing aspect is that the high level of mortality is attributed to lack of awareness. Early diagnosis with aggressive treatment can lead to successful prognosis for the patient. Here, we describe a brief overview of the current understanding of the pathophysiology of GAE, available diagnostic methods, possible therapeutic interventions and the causative agents.
\end{abstract}

Key words: Granulomatous amoebic encephalitis (GAE), clinical diagnosis, preventative measures, Acanthamoeba, Balamuthia mandrillaris

\section{INTRODUCTION}

Granulomatous amoebic encephalitis (GAE) is a serious disease leading almost always to death. It can be caused by two protozoan pathogens, Acanthamoeba and Balamuthia mandrillaris. GAE has become increasingly important in the recent years. This is due to i) the increasing numbers of immunocompromised patients, ii) the excessive use of antibiotics, iii) the availability of improved diagnostic methods and iv) global warming with increased outdoor activities adding to the ubiquity of these pathogens, thus increased exposure to the susceptible hosts. With the growing HIV pandemic, it is reasonable to predict further increase in the numbers of opportunistic infections. Among other opportunistic pathogens, Acanthamoeba and B. mandrillaris have remained a significant threat to human health and gained particular attention in recent years. The purpose of this review is to provide our current understanding of the disease and the infectious agents, diagnostic methods and possible therapeutic interventions.

The disease: Although GAE is a rare infection, it almost always proves fatal. The mechanisms associated with the pathogenesis remain unclear; however the pathophysiological complications involving the central nervous system (CNS) most likely include induction of the pro-inflammatory responses, invasion of the bloodbrain barrier and the connective tissue and neuronal damage leading to the brain dysfunction. The routes of entry include lower respiratory tract leading to amoebae invasion of the intravascular space followed by the haematogenous spread. Skin lesions may provide direct amoebae entry into the bloodstream, thus bypassing the lower respiratory tract (Fig. 1). Amoebae entry into the
CNS most likely occurs at the sites of the blood-brain barrier ${ }^{[1,2]}$. The cutaneous and respiratory infections can last for months but the involvement of the CNS can result in fatal consequences within days or weeks. The clinical picture may resemble viral, bacterial or tuberculosis meningitis. For example, the clinical symptoms involve headache, fever, behavioral changes, hemiparesis, lethargy, stiff neck, aphasia, ataxia, vomiting, nausea, cranial nerve palsies, increased intracranial pressure, confused state, seizures and finally leading to death. The term "granulomatous" indicates hemorrhagic necrotizing lesions or brain abscess (detected by neuroimaging scans) with severe meningeal irritation and encephalitis ${ }^{[2,3]}$. Patients with respiratory infections, skin ulcerations or brain abscesses should be suspected for infections due to Acanthamoeba and B. mandrillaris. Post mortem examination often shows severe edema and hemorrhagic necrosis. It is not known whether this necrotic phase is caused by actively feeding trophozoites or inflammatory processes such as the release of cytokines. The lesions due to GAE are most numerous in the basal ganglia, midbrain, brainstem and cerebral hemiparesis with characteristic lesions in the CNS parenchyma resulting in granulomatous encephalitis. The granuloma is composed of CD4, CD8, B-lymphocytes, plasma cells and macrophages. Granulomatous response may be absent or minimal in patients with a severely impaired immune system that is interpreted as impairment of the cellular immune response $^{[4]}$. The affected organs other than the CNS may include subcutaneous tissue, skin, liver, lungs, kidneys, pancreas, prostate, lymph nodes and bone marrow. The predisposing factors in contracting GAE are not clearly understood. Although the majority of GAE cases due to Acanthamoeba are limited to

Corresponding Author: Naveed Ahmed Khan, School of Biological and Chemical Sciences, Birkbeck College, University of London, London, WC1E 7HX, England, UK, Tel: 44-(0)207-079-0797. Fax: 44-(0)207-631-6246 
individuals with a weakened immune system, $B$. mandrillaris infections have been shown to occur in relatively immunocompetent people. This is shown with the findings that GAE can develop in individuals with no history of syphilis, diabetes mellitus, malignancies, fungal and mycobacterial infections and in patients negative for HIV-1 and HIV $-2^{[5]}$. However, patients suffering from these as well as other diseases including lymphoproliferative disorders, hematologic disorders, pneumonitis, renal failure, liver cirrhosis, rhinitis, pharyngitis, gammalobulinemia, systemic lupus erythematosus, glucose-6-phosphate deficiency, tuberculosis, chronically alcoholic, radiotherapy, malnourished, chronically ill, or debilitated, are particularly at risk. Patients undergoing organ/tissue transplantation with immunosuppressive therapy, steroids and excessive antibiotics are also at risk of contracting GAE (Fig. 2). Future studies will determine the precise host factors contributing to this fatal infection, which may help identify preventative and therapeutic measures.

Clinical diagnosis of GAE: As indicated above, GAE is a rare infection with very poor prognosis. Early diagnosis with aggressive treatment is crucial for a successful outcome for the patient. GAE symptoms are similar to other CNS pathogens including virus, bacteria and fungi. This makes GAE diagnosis problematic and requires very high suspicion and expertise. The brain image analyses using computed tomography (CT) or magnetic resonance imaging (MRI) scan may show multifocal areas of signal intensities or lesions indicating brain abscesses or tumours suggestive of the CNS defects. The cerebrospinal fluid (CSF) findings, although not confirmatory of GAE, are of value in diagnosing the CNS involvement. Pleocytosis with lymphocytic predominance is an important feature with elevated polymorphonuclear leukocytes, increased protein concentrations, decreased glucose concentrations and minimal cloudiness ${ }^{[5]}$. The absence of viral and bacterial pathogens should be strongly suspected of GAE. Due to the low density of parasites, the detection of host immune response should be attempted primarily. The demonstration of high levels of Acanthamoeba- or B. mandrillaris-specific antibodies in a patient's serum may provide a useful and straightforward method to further suspect GAE infection. This is performed using indirect immunofluorescence (IIF) assays. The serial dilutions of the patient's serum are incubated with fixed amoebae-coated slides, followed by incubation with fluorescein isothiocyanate (FITC)-labelled anti-human antibody and visualized under fluorescent microscope. It is important to remember that the levels of antiamoebae antibodies in normal populations may be in the range of $1: 20$ to $1: 60$. However, patients with a severely impaired immune system may not develop a high titre, thus other clinical findings should be taken into account for the correct diagnosis.

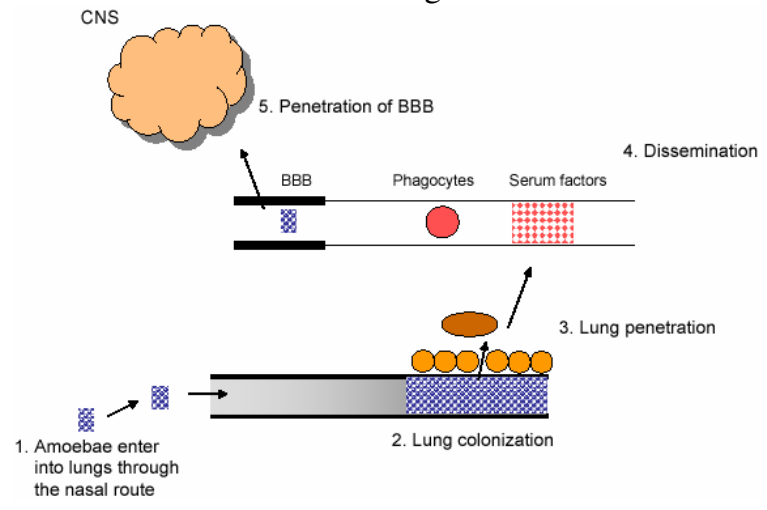

Fig. 1: The model of granulomatous amoebic encephalitis. Amoebae enter lungs via the nasal route. Next, amoebae traverse the lungs into the bloodstream (or directly through skin lesions), followed by haematogenous spread. Finally, amoebae cross the blood-brain barrier and enter into the central nervous system (CNS) to produce disease. The olfactory neuroepithelium may provide an alternative route of entry into the CNS

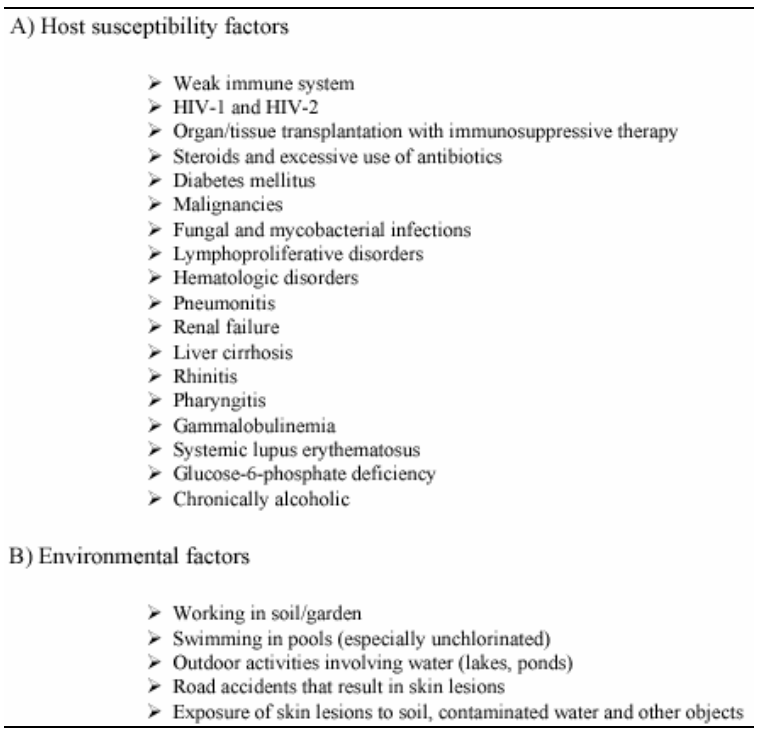

Fig. 2: The risk factors associated with granulomatous amoebic encephalitis. A) Host susceptibility factors and $\mathrm{B}$ ) environmental risk factors to $\mathrm{A}$

The confirmatory evidence comes from direct microscopic observation of amoebae in the CSF (after centrifugation at low speed) or in the brain biopsy but requires familiarity of morphological characters. Giemsa-Wright, acridine orange or calcofluor white staining may facilitate morphological-based positive identification of these amoebae. The lack of familiarity with amoebae morphological characteristics may require immunohistochemical studies using antisera 
made against Acanthamoeba and B. mandrillaris to identify the etiologic agent, which should aid in the clinical diagnosis of GAE.

In addition, it is helpful to inoculate a portion of the CSF and/or brain biopsy for amoebae culturing. For Acanthamoeba, the clinical specimens can be inoculated onto non-nutrient agar plate seeded with Gram-negative bacteria. Acanthamoeba feeds on bacteria as food source and depending on the number of amoebae in the specimen, trophozoites can be observed within a few hours (up to $12 \mathrm{~h}$ ). However in the absence of amoebae, plates should be monitored for up to 7 days $^{[6,7]}$. This method is particularly useful if problems are encountered in differentiating Acanthamoeba from monocytes, polymorphonuclear leukocytes and macrophages. Balamuthia mandrillaris do not feed on Gram-negative bacteria and should be inoculated onto mammalian cell cultures but it may take up to several weeks $^{[8]}$. Alternatively, PCR-based methods have been developed for the clinical diagnosis of amoebae infections and provide rapid diagnosis from the clinical specimens ${ }^{[8,9]}$.

Treatment: For GAE infection, there are no recommended treatments and the majority of cases are identified at the post-mortem stage. This is due to i) delayed diagnosis, ii) low sensitivity of amoebae to many anti-amoebic agents and iii) the limited efficacy of anti-amoebic agents to cross the blood-brain barrier into the central nervous system. Current therapeutic agents include a combination of drugs including ketoconazole, fluconazole, sulfadiazine, pentamidine isethionate, fluorcytosine, amphotericin B, trifluorperazine or thiroridizine, clarithromycin, azithromycin, itraconazole, or rifampin that may be effective against CNS infections due to free-living amoebae but have severe side effects. Recent studies have suggested that alkylphosphocholine compounds, such as hexadecylphosphocholine exhibit anti-amoebic properties as well as cross the blood-brain barrier, thus may have value in the treatment of $\mathrm{GAE}^{[10,11]}$. Even with treatment, survivors may develop disability such as hearing loss, vision impairment etc.

The infectious agents: Both Acanthamoeba and $B$. mandrillaris are protozoan pathogens and are considered free-living amoebae. They are widely distributed in the environment. Acanthamoeba were first observed in yeast (Cryptococcus) cultures by Castellani $^{[12]}$ and Volkonsky created genus Acanthamoeba for these amoebae ${ }^{[13]}$. The name acanth (Greek: meaning spikes) was added to these amoebae due to the presence of spine-like structures on the surface of these organisms (Fig. 3). For the next two decades, these organisms were largely ignored. However, in the late 1950s, they were discovered as tissue culture contaminants ${ }^{[14,15]}$. Later, Culbertson et $a l .{ }^{[14,16]}$ demonstrated, for the first time, the pathogenic potential of these organisms by exhibiting their ability
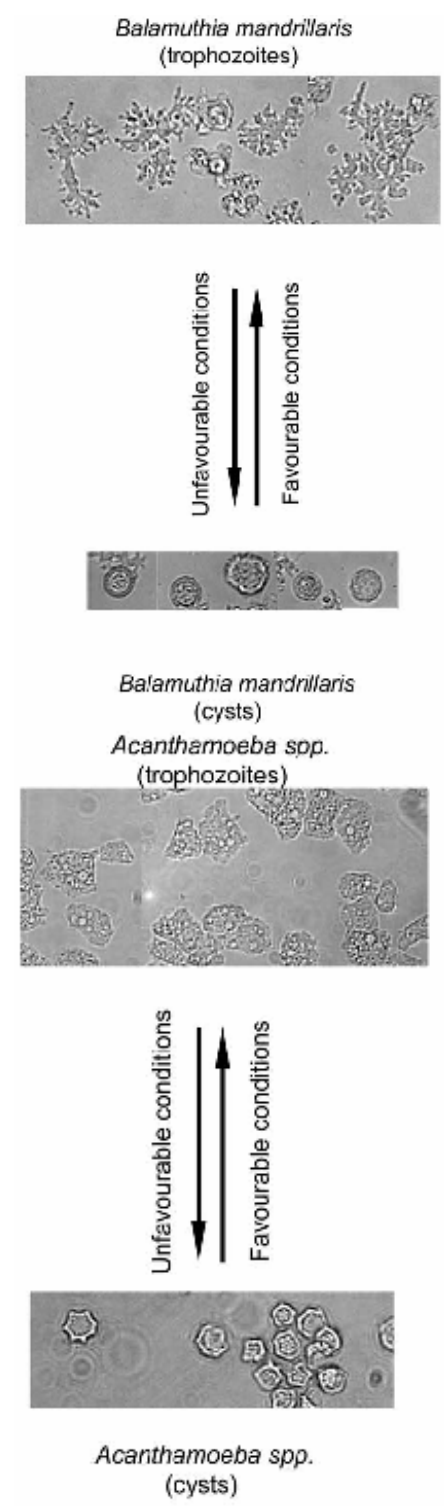

Fig. 3: The life cycle of Acanthamoeba and Balamuthia mandrillaris. Infective form of amoebae is known as trophozoites. Under unfavorable conditions, trophozoites differentiate into round cysts. X250

to produce cytopathic effects on monkey kidney cells in vitro and to kill laboratory animals, i.e., monkeys in vivo. The first clearly identified GAE due to Acanthamoeba was observed in $1972^{[17]}$. Soon after, Acanthamoeba was identified as a causative agent of blinding keratitis ${ }^{[18]}$ and then clearly associated with Legionairre's disease by harbouring Legionella pneumophila $^{[19]}$.

In contrast to Acanthamoeba, B. mandrillaris has only been recently discovered in 1986 from the brain of a baboon who died of meningoencephalitis and were described as a new genus, i.e., Balamuthia ${ }^{[20,21]}$. Later, B. mandrillaris were associated with causing GAE in 
$1991^{[22,23]}$. Since then, more than 100 cases of GAE due to $B$. mandrillaris have been identified. Based on $16 \mathrm{~S}$ rRNA gene sequences, it is determined that $B$. mandrillaris is closely related with Acanthamoeba ${ }^{[9,24,25]}$.

The life cycle of both these pathogens undergoes two stages, a vegetative trophozoite stage and a resistant cyst stage (Fig. 3). The trophozoites are normally in the range of $15-45 \mu \mathrm{m}$ in diameter, however the size vary significantly between different species. The cell division is asexual and occurs by binary fission. Under harsh conditions (lack of food, extremes in osmolarity, $\mathrm{pH}$ and temperatures), amoebae switch into resistant cyst stage. In simple terms, trophozoite becomes metabolically inactive (minimal metabolic activity), excess food, water and particulate matter is expelled and the trophozoite encloses itself within a resistant shell to survive harsh periods. The cellular levels of RNA, proteins, triacylglycerides and glycogen declines substantially during the encystment process resulting in decreased cellular volume and dry weight. The cyst stage is approximately $10-20 \mu \mathrm{m}$. The trophozoites emerge from the cysts under favourable conditions leaving behind the outer shell and actively reproduce, thus completing the cycle.

\section{CONCLUSION}

Despite advances in the antimicrobial chemotherapy and supportive care, GAE infections have remained significant. This is due to our incomplete understanding of the pathogenesis and pathophysiology of GAE infections. Successful treatment requires increased awareness and strong suspicion based on clinical findings, early diagnosis followed by aggressive treatment using a mixture of drugs. With the HIV pandemic and other contributing factors, there is a clear increase in the number of susceptible hosts presenting a threat of free-living amoebae to human health. In addition, warmer climates will undoubtedly add to the increased ubiquity of these pathogens and thus increased exposure to the susceptible hosts. Thus there is an urgent need to our understanding of amoebae pathogenesis both at the molecular- and cellular-level as well as their ability to transmit, adapt to diverse conditions, overcome host barriers and emerge as infective trophozoite to produce infection will provide targets for therapeutic interventions and to design strategies for preventative measures.

\section{ACKNOWLEDGEMENTS}

This work is supported by grants from the Faculty Research Fund, University of London, The Nuffield Foundation and The Royal Society.

\section{REFERENCES}

1. Martinez, A.J., 1991. Infections of the central nervous system due to Acanthamoeba. Rev. Infect. Dis., 13: S399-S402.

2. Martinez, A.J. and G.S. Visvesvara, 1997. Freeliving, amphizoic and opportunistic amebas. Brain Pathol., 7: 583-598.

3. Martinez, A.J., 1985. Free-living Amebas: Natural History, Prevention, Diagnosis, Pathology and Treatment of Disease. CRC Press, Boca Raton, Florida, pp: 156.

4. Martinez, M.S., G. Gonzalez-Mediero, P. Santiago, A. Rodriguez de Lope, J. Diz, C. Conde and G.S. Visvesvara, 2000. Granulomatous amebic encephalitis in a patient with AIDS: Isolation of Acanthamoeba sp. Group II from brain tissue and successful treatment with sulfadiazine and fluconazole. J. Clin. Microbiol., 38: 3892-3895.

5. Marciano-Cabral, F. and G. Cabral, 2003. Acanthamoeba spp. as agents of disease in humans. Clin. Microbiol. Rev., 16: 273-307.

6. Khan, N.A., E.L. Jarroll and T.A. Paget, 2002. Molecular and physiological differentiation between pathogenic and non-pathogenic Acanthamoeba. Curr. Microbiol., 45: 197-202.

7. Khan, N.A., 2003. Pathogenesis of Acanthamoeba infections. Microb. Pathogen., 34: 277-285.

8. Jayasekera, S., A. Matin, J. Sissons, A.H. Maghsood and N.A. Khan, 2005. Balamuthia mandrillaris stimulates interleukin-6 release in primary human brain microvascular endothelial cells via a phosphatidylinositol 3-kinase-dependent pathway. Microb. Infect., 7: 1345-1351.

9. Booton, G.C., J.R. Carmichael, G.S. Vivesvara, T.J. Byers and P.A. Fuerst, 2003. Identification of Balamuthia mandrillaris by PCR assay using the mitochondrial 16S rRNA gene as a target. J. Clin. Microbiol., 41: 453-455.

10. Kotting, J., M.R. Berger, C. Unger and H. Eibl, 1992. Alkylphosphocholines: influence of structural variations on biodistribution of antineoplastically active concentrations. Cancer Chemother. Pharmacol., 30: 105-112.

11. Walochnik, J., M. Duchene, K. Seifert, A. Obwaller, T. Hottkowitz, G. Wiedermann, H. Eibl and H. Aspock, 2002. Cytotoxic activities of alkylphosphocholines against clinical isolates of Acanthamoeba spp. Antimicrob. Agents Chemother., 46: 695-701.

12. Castellani, A., 1930. An amoeba found in culture of yeast: Preliminary note. J. Trop. Med. Hyg., pp: 160.

13. Volkonsky, M., 1931. Hartmanella castellanii Douglas, et classification des hartmannelles. Arch. Zool. Exp. Gen., 72: 317-339. 
14. Culbertson, C.G., J.W. Smith and J.R. Miner, 1958. Acanthamoeba: Observation on animal pathogenicity. Science, 127: 1506.

15. Jahnes, W.G., H.M. Fullmer and C.P. Li, 1957. Free-living amoebae as contaminants in monkey kidney tissue culture. Proc. Soc. Exp. Biol. Med., 96: 484-488.

16. Culbertson, C.G., J.W. Smith, H.K. Cohen and J.R. Miner, 1959. Experimental infection of mice and monkeys by Acanthamoeba. Am. J. Pathol., 35: 185-197.

17. Jager, B.V. and W.P. Stamm, 1972. Brain abscesses caused by free-living amoeba probably of the genus Hartmannella in a patient with Hodgkin's disease. Lancet, 2: 1343-1345.

18. Nagington, J., P.G. Watson, T.J. Playfair, J. McGill, B.R. Jones and A.D. Steele, 1974. Amoebic infections of the eye. Lancet, 2: 1537-1540.

19. Rowbotham, T.J., 1980. Preliminary report on the pathogenicity of Legionella pneumophila for freshwater and soil amoebae. J. Clin. Pathol., 33: 1179-1183.

20. Visvesvara, G.S., A.J. Martinez, F.L. Schuster, G.J. Leitch, S.V. Wallace, T.K. Sawyer and M. Anderson, 1990. Leptomyxid ameba, a new agent of amebic meningoencephalitis in humans and animals. J. Clin. Microbiol., 28: 2750-2756.
21. Visvesvara, G.S., F.L. Schuster and A.J. Martinez, 1993. Balamuthia mandrillaris, N.G., N. Sp., agent of amebic meningoencephalitis in humans and other animals. J. Eukaryot. Microbiol., 40: 504514.

22. Anzil, A.P., C. Rao, M.A. Wrzolek, G.S. Visvesvara, J.H. Sher and P.B. Kozlowski, 1991. Amebic meningoencephalitis in a patient with AIDS caused by a newly recognized opportunistic pathogen. Leptomyxid ameba. Arch. Pathol. Lab. Med., 115: 21-25.

23. Taratuto, A.L., J. Monges, J.C. Acefe, F. Meli, A. Parades and A.J. Martinez, 1991. Leptomyxid amoeba encephalitis: Report of the first case in Argentina. Trans. R. Soc. Trop. Med. Hyg., 85: 77.

24. Amaral Zettler, L.A., T.A. Nerad, C.J. O'Kelly, M.T. Peglar, P.M. Gillevet, J.D. Silberman and M.L. Sogin, 2000. A molecular reassessment of the leptomyxid amoebae. Protist, 151: 275-282.

25. Booton, G.C., J.R. Carmichael, G.S. Vivesvara, T.J. Byers and P.A. Fuerst, 2003. Genotyping of Balamuthia mandrillaris based on nuclear 18S and mitochondrial 16S rRNA genes. Am. J. Trop. Med. Hyg., 68: 65-69. 